\title{
Objective Sedentary Time, Moderate-to- Vigorous Physical Activity, and Physical Capability in a British Cohort
}

\author{
VICTORIA L. KEEVIL ${ }^{1}$, ANDREW J. M. COOPER ${ }^{2}$, KATRIEN WIJNDAELE ${ }^{2}$, ROBERT LUBEN ${ }^{1}$, \\ NICHOLAS J. WAREHAM ${ }^{2}$, SOREN BRAGE ${ }^{2}$, and KAY-TEE KHAW ${ }^{1}$ \\ ${ }^{1}$ Department of Public Health and Primary Care, Strangeways Research Laboratory, University of Cambridge, Cambridge, \\ UNITED KINGDOM; and ${ }^{2}$ MRC Epidemiology Unit, Institute of Metabolic Science, University of Cambridge, Addenbrookes \\ Biomedical Campus, Cambridge, UNITED KINGDOM
}

\begin{abstract}
KEEVIL, V. L., A. J. M. COOPER, K. WIJNDAELE, R. LUBEN, N. J. WAREHAM, S. BRAGE, AND K.-T. KHAW. Objective Sedentary Time, Moderate-to-Vigorous Physical Activity, and Physical Capability in a British Cohort. Med. Sci. Sports Exerc., Vol. 48, No. 3, pp. 421-429, 2016. Purpose: Sedentariness has been proposed as an independent risk factor for poor health. However, few studies have considered associations of sedentary time (ST) with physical functional health independent of time spent in moderate-to-vigorous physical activity (MVPA). Methods: Community-based men and women ( $n=8623,48-92$ yr old) in the European Prospective Investigation of Cancer-Norfolk study attended a health examination for objective measurement of physical capability, including grip strength (Smedley dynamometer (kg)), usual walking speed (UWS $\left(\mathrm{cm}^{-1} \mathrm{~s}^{-1}\right)$ ), and timed chair stand speed (TCSS (stands per minute)). Of these, 4051 participants wore an accelerometer (GT1M ActiGraph) for $7 \mathrm{~d}$ to estimate time spent in MVPA (MVPA, $\geq 1952$ counts per minute) and ST (ST, $<100$ counts per minute). Relations between physical capability outcomes and both MVPA and ST were explored using linear regression. The mutual independence of associations was also tested, and ST-MVPA interactions were explored using fractional polynomial models to account for nonlinear associations. Results: Men in the highest compared with those in the lowest sex-specific quartile of MVPA were stronger (1.84 kg; 95\% confidence interval (CI), 0.79-2.89), had faster UWS (11.7 $\mathrm{cm} \cdot \mathrm{s}^{-1} ; 95 \%$ CI, 8.4-15.1) and faster TCSS (2.35 stands per minute; $95 \%$ CI, 1.11-3.59) after multivariable adjustment. Similarly, women in the highest quartile of MVPA were stronger (2.47 kg; 95\% CI, 1.79-3.14) and had faster UWS (15.5 $\mathrm{cm}^{-1} \mathrm{~s}^{-1} ; 95 \%$ CI, 12.4-18.6) and faster TCSS (3.27 stands per minute; $95 \%$ CI, 2.19-4.25). Associations persisted after further adjustment for ST. Associations between higher ST and lower physical capability were also observed, but these were attenuated after accounting for MVPA. Furthermore, no MVPA-ST interactions were observed $\left(P_{\text {interactions }}>0.05\right)$. Conclusions: More time spent in MVPA was associated with higher physical capability, but there were no independent ST associations. Key Words: ACCELEROMETRY, OBJECTIVE ASSESSMENT, AGING, PHYSICAL FUNCTION
\end{abstract}

Address for correspondence: Victoria L Keevil, B.M. B.Ch., EPIC, Strangeways Research Laboratory, Wort's Causeway, Cambridge CB1 8RN, United Kingdom; E-mail: vlk20@cam.ac.uk.

Submitted for publication February 2015.

Accepted for publication September 2015.

Supplemental digital content is available for this article. Direct URL citations appear in the printed text and are provided in the HTML and PDF versions of this article on the journal's Web site (www.acsm-msse.org).

0195-9131/16/4803-0421/0

MEDICINE \& SCIENCE IN SPORTS \& EXERCISE $_{\circledast}$

Copyright $(2015$ by the American College of Sports Medicine. This is an open access article distributed under the Creative Commons Attribution License 4.0 (CCBY), which permits unrestricted use, distribution, and reproduction in any medium, provided the original work is properly cited.

DOI: 10.1249/MSS.0000000000000785
$\mathrm{P}$ hysical capability gradually declines during old age, with many older adults now reaching thresholds associated with disability before death. Therefore, interventions to prevent or delay this decline offer the potential to improve quality of life and decrease health care expenditure. One line of inquiry is the functional benefit associated with increasing physical activity (PA).

Public health guidelines already recommend that older adults ( $>60 \mathrm{yr}$ old) undertake $\geq 150 \mathrm{~min} \cdot \mathrm{wk}^{-1}$ of moderateto vigorous-intensity PA (MVPA) and muscle strengthening exercises more than twice a week and reduce sedentariness (9), on the basis of evidence considering a range of health outcomes $(18,22,34)$. However, although a number of studies have reported associations between higher PA and better 
physical functional health (29), not all results concur. Inconsistency particularly occurs when objective markers of physical capability rather than self-reported disability are the outcome measures of interest, e.g., grip strength or usual walking speed (UWS). Some studies report positive associations $(33,39)$, whereas others do not or find associations that differ by sex $(4,6,24,25,28,37)$.

Most of these previous studies have used questionnairebased methods to assess PA $(4,6,25,28,29,33,37,39)$, which are subject to reporting biases and random measurement error, e.g., due to difficulties with recall (17). In addition, PA questionnaires (PAQ) are only sensitive enough to distinguish broad categories of MVPA, e.g., low, moderate, or high. Thus, the dose-response relation between MVPA and functional health remains unclear, e.g., whether there is a "dose" below which MVPA is ineffective (29). This is an important question, given that many older adults do not achieve the recommended amounts of MVPA (38).

There is also paucity of evidence relating sedentary behavior, defined as time awake spent sitting or lying when energy expenditure is low $(\leq 1.5$ times the resting energy expenditure), with physical functional health. We previously reported an association between more time spent watching television, a common leisure time sedentary behavior, and lower UWS, which persisted when television viewing time was measured prospectively (21). However, it is unclear whether associations with sedentary behaviors such as television viewing time reflect the physiological consequences of prolonged sitting or are explained by other confounding factors associated with these behaviors (14). Among the population-based studies considering sedentariness and physical functional health, only one measured physical function, PA, and sedentary time objectively. This study in older Portuguese adults reported independent associations between higher scores on a physical performance battery and both greater volumes of objectively measured MVPA and lower volumes of sedentary time (32). However, the cohort used in this study was small and sedentary time-MVPA interactions were not explored.

Therefore, we aimed to investigate the independent doseresponse associations of objectively measured sedentary time and MVPA with objectively measured physical capability in a large population-based sample of middle-age and older British men and women. We also examined whether interactions between sedentary time and MVPA were evident with respect to the outcomes considered.

\section{METHODS}

Study population. The European Prospective Investigation of Cancer (EPIC) - Norfolk study enroled more than 25,000 community-based men and women at baseline (1993-1997), who were age 40-79 yr and registered with a participating general practitioner in and around the city of Norwich (Norfolk, United Kingdom). The full details of the study design and follow-up of participants have been reported previously (15). At the third health examination (3HC; 2006-2011), 8623 participants now age 48-92 yr attended a central research clinic in Norwich and underwent measurement of physical capability. A written informed consent was obtained at baseline and renewed at the $3 \mathrm{HC}$. The study complies with the principles of the Declaration of Helsinki, and ethical approval was given by the Norfolk local research ethics committee and the East Norfolk and Waveney National Health Service Research Governance Committee.

PA. At the $3 \mathrm{HC}$ clinic visit, participants were also invited to wear an accelerometer (ActiGraph GT1M model; ActiGraph LLC, Pensacola, FL) for seven consecutive days on an elastic belt at the level of the right hip during all waking hours except when bathing, showering, or swimming. This monitor is a uniaxial accelerometer, which measures the vertical (when standing up) acceleration (counts) in 5-s, epochs thereby quantifying the intensity of movement. Accelerometry records were screened for continuous strings of zero acceleration lasting $\geq 90 \mathrm{~min}$, which were considered as monitor nonwear time. Participants with less than four valid wear days (each containing $>600 \mathrm{~min}$ of valid wear time) were excluded $(n=118)$. In addition, it was not possible to invite all participants to wear an activity monitor because the monitors were not always available over the $6 \mathrm{yr}$ of the clinic duration. All in all, this left 4051 participants with valid accelerometry data. Sedentary time was defined as time spent at $<100$ counts per minute (16) and MVPA as time spent at $\geq 1952$ counts per minute (10). All time spent in MVPA was included, and it was not restricted to MVPA accumulated in 10-min bouts.

Physical capability. The physical capability measures used in this study are feasible and reliable measures that have been validated in other epidemiological studies and predict future health outcomes $(3,13)$. In addition, grip strength and UWS form part of the National Institutes of Health toolbox, created to standardize functional measurements in research studies (www.nihtoolbox.org). Maximum grip strength $(\mathrm{kg})$ was measured using a Smedley dynamometer (Scandidact, Kvistgaard, Denmark) while the participant was standing with their forearm bent at $90^{\circ}$. Participants who were unable to stand performed the test sitting. The maximum strength achieved from two recordings in each hand, alternating measurement between hands, was used. UWS was measured as time taken to walk $4 \mathrm{~m}$ at a comfortable pace using a walking aid if necessary. Participants undertook two walks, and the course distance was divided by the average time taken to estimate UWS $\left(\mathrm{cm} \cdot \mathrm{s}^{-1}\right)$. Time to complete five chair rises was measured with participants seated in a straightbacked chair, with their feet flat on the floor and arms folded across the chest. Participants were timed from the command "stand" to the end of the fifth rise, and timed chair stand speed (TCSS) was calculated (stands per minute, $60 \times(5 /$ time (s))). Participants who could not stand from a chair unaided were excluded.

Covariables. The day and month on which the 3HC clinic appointment was held (time of year) were recorded. At 
the $3 \mathrm{HC}$ clinic, weight and height were measured with the participant wearing light clothing using digital scales (Tanita) and a stadiometer (Chasmores, United Kingdom).

Participants self-reported their smoking status (current, ex-smokers, or never smokers), current wealth (more than enough, just enough, or not enough money), and alcohol intake (units per week). Comorbidity was estimated by combining self-reported stroke, cancer, diabetes, and/ or myocardial infarction (MI) (obtained at the 1HC and 2HC), with incident data captured over the follow-up period (via record linkage with hospital episode statistics). Comorbidity was included as a binary variable in analyses (yes/no).

Statistical analyses. Participant characteristics were described using means (SD), medians (interquartile range and frequencies $(\%, n)$. The characteristics of those included in the analytical sample versus those excluded (because of missing accelerometry data) were compared using $t$-tests and chi-square tests. Associations between sedentary time, MVPA, and physical capability were examined using linear regression.

First, sex-specific quartiles (Q) of sedentary time and MVPA were defined and associations with physical capability were examined in separate models for each exposure measure. The highest quartile of sedentary time and the lowest quartile of MVPA were used as the referent categories (in both cases henceforth referred to as Q1) so that regression coefficients $(95 \%$ confidence intervals $(\mathrm{CI})$ ) were the differences in grip strength, UWS, and TCSS associated with lower amounts of sedentary time and higher amounts of MVPA. Associations were adjusted on the basis of a priori reason for age and monitor wear time (model 1) and then additionally for height, weight, current wealth, comorbidity, smoking status, and alcohol intake (model 2). Because activity monitors were only worn during one $7-\mathrm{d}$ period, the United Kingdom has a temperate climate, and weather conditions are known to correlate to PA levels (36), model 2 was also adjusted for the time of year when the accelerometer was worn. Tests for trend were examined by entering exposure quartiles into the models without identifying them as categorical variables $\left(P_{\text {trend }}\right)$. Deviation from linearity was assessed by comparing models with exposure quartiles entered as categorical versus linear predictor variables using likelihood ratio tests $\left(P_{\text {linearity }}\right)$. Finally, to establish whether associations between physical capability and either sedentary time or MVPA were mutually independent, both exposure variables were included together in sex-specific regression models.

To explore possible sedentary time-MVPA interactions, associations between physical capability and sedentary time were subsequently examined by quartile of MVPA. To optimize power, both sexes were combined and MVPA quartiles were generated for the whole cohort. In addition, sedentary time was explored as a continuous exposure measure $\left(h \cdot d^{-1}\right)$.

Then, sedentary time $\left(\mathrm{h} \cdot \mathrm{d}^{-1}\right)$ and MVPA $\left(\mathrm{min} \cdot \mathrm{d}^{-1}\right)$ were included together in multivariable fractional polynomial models to formally test interaction between the two exposures. Fractional polynomial models were used to account for the potentially nonlinear associations between the exposures and physical capability because spurious interactions can be observed if linearity is incorrectly assumed. Interactions between sedentary time and MVPA were evaluated using likelihood ratio tests comparing models with and without the interaction terms using a technique developed by Royston and Sauerbrei (31). To increase model stability, the highest values of MVPA were recoded to $90 \mathrm{~min} \cdot \mathrm{d}^{-1}$, and all values of MVPA were divided by 30 . In addition, analyses were repeated after stratifying the cohort into young (participants age $<70 \mathrm{yr}$ ) and older (participants age $\geq 70 \mathrm{yr}$ ) age groups.

All analyses were conducted using Stata version 12.0 (StataCorp LP, College Station, TX).

Sensitivity analyses. In addition, the independent associations of both MVPA and sedentary time with physical capability, as well as possible interactions between them, were evaluated with accelerometer data averaged over 60 -s epochs.

\section{RESULTS}

Participants who did not wear an activity monitor or have at least $4 \mathrm{~d}$ of valid data $(n=4572)$ were similar in terms of body mass index, self-reported PA, and wealth to those who did $(n=4051)$ (see Document, Supplemental Digital Content, Table 1, EPIC-Norfolk participants who attended the $3 \mathrm{HC}$ and did or did not have at least $4 \mathrm{~d}$ of objectively measured PA, http://links.lww.com/MSS/A583). There was no difference in age between the two groups for women and only a slight difference in age for men.

Participants with missing covariable data were also excluded from analyses (weight, $n=2$; height, $n=6$; current wealth, $n=219$; alcohol intake, $n=169$ ). Final sample sizes (both sexes combined) ranged from 3340 to 3691 depending on the physical capability measure examined $(n=40$ missing grip strength, $n=35$ missing UWS, and $n=386$ missing TCSS). The characteristics of the men and women who had complete data and participated in this study are shown in Table 1.

On average, the 1674 men and 2052 women included in this study wore the accelerometer for $882 \mathrm{~min} \cdot \mathrm{d}^{-1}$ (SD, $70.5)$ and $864 \mathrm{~min} \cdot \mathrm{d}^{-1}$ (SD, 64.7), respectively. Participants

TABLE 1. The characteristics of participants included in this study.

\begin{tabular}{lcc}
\hline Covariable & Men $(\boldsymbol{n}=\mathbf{1 6 7 4})$ & Women $(\boldsymbol{n}=\mathbf{2 0 5 2})$ \\
\hline Mean $(\mathrm{SD})$ & & \\
Age $(\mathrm{yr})$ & $69.8(7.6)$ & $68.0(7.5)$ \\
Height $(\mathrm{cm})$ & $173.5(6.6)$ & $160.6(6.2)$ \\
$\quad$ Weight $(\mathrm{cm})$ & $81.6(12.0)$ & $68.5(12.8)$ \\
Frequency, \% $(n)$ & & \\
$\quad$ Current wealth & & \\
$\quad$ More than enough money & $30.6(513)$ & $27.0(554)$ \\
Just enough money & $63.3(1059)$ & $67.4(1384)$ \\
Not enough money & $6.0(102)$ & $5.6(114)$ \\
Smoking status & $39.6(663)$ & $58.7(1205)$ \\
Never smoked & $56.4(944)$ & $37.0(760)$ \\
Ex-smoker & $4.0(67)$ & $4.2(87)$ \\
$\quad$ Current smoker & & \\
$\quad$ Comorbidity & $19.1(319)$ & $16.6(341)$ \\
$\quad$ Yes & & \\
Median, (interquartile range) & $6(1-12)$ & $2(0-7)$ \\
$\quad$ Alcohol intake (units per week) & & \\
\hline
\end{tabular}


TABLE 2. Differences in physical capability by quartile of MVPA.

\begin{tabular}{|c|c|c|c|c|c|c|c|c|c|}
\hline \multirow[b]{2}{*}{ MVPA (Quartiles) } & \multicolumn{3}{|c|}{ Difference in Grip Strength $(95 \% \mathrm{CI})(\mathrm{kg})$} & \multicolumn{3}{|c|}{ Difference in UWS $(95 \% \mathrm{CI})\left(\mathrm{cm} \cdot \mathrm{s}^{-1}\right)$} & \multicolumn{3}{|c|}{ Difference in TCSS $(95 \% \mathrm{CI})$ (stands per minute) } \\
\hline & $n$ & Model 1 & Model 2 & $n$ & Model 1 & Model 2 & $n$ & Model 1 & Model 2 \\
\hline Men & 1664 & & & 1662 & & & 1506 & & \\
\hline Q1 (least) & & 0 & 0 & & 0 & 0 & & 0 & 0 \\
\hline Q2 & & $0.88(-0.09$ to 1.85$)$ & $1.13(0.18$ to 2.08$)$ & & $10.4(7.4$ to 13.4$)$ & $9.3(6.3$ to 12.3$)$ & & 1.69 (0.53 to 2.84$)$ & $1.61(0.46$ to 2.76$)$ \\
\hline Q3 & & $1.83(0.82$ to 2.83$)$ & $2.21(1.21$ to 3.21$)$ & & $13.6(10.5$ to 16.7$)$ & $11.6(8.4$ to 14.8$)$ & & 2.16 (0.98 to 3.35$)$ & $2.09(0.84$ to 3.23$)$ \\
\hline Q4 (most) & & 1.26 (0.22 to 2.30$)$ & 1.84 (0.79 to 2.89$)$ & & $14.2(10.9$ to 17.4$)$ & 11.7 (8.4 to 15.1$)$ & & $2.43(1.22$ to 3.64$)$ & 2.35 (1.11 to 3.59$)$ \\
\hline$P_{\text {trend }}$ & & & $<0.001$ & & & $<0.001$ & & & $<0.001$ \\
\hline$P_{\text {linearity }}$ & & & 0.05 & & & $<0.001$ & & & 0.21 \\
\hline Women & 2022 & & & 2029 & & & 1834 & & \\
\hline Q1 (least) & & 0 & 0 & & 0 & 0 & & 0 & 0 \\
\hline Q2 & & 1.18 (0.56 to 1.79$)$ & 1.38 (0.78 to 1.98$)$ & & 11.8 (9.1 to 14.6$)$ & 9.8 (7.1 to 12.5$)$ & & 1.54 (0.54 to 2.55$)$ & $1.51(0.52$ to 2.50$)$ \\
\hline Q3 & & 0.92 (0.28 to 1.55$)$ & $1.22(0.58$ to 1.85$)$ & & 15.9 (13.0 to 18.8$)$ & 12.1 (9.2 to 15.0$)$ & & 2.97 (1.93 to 4.00$)$ & 2.68 (1.64 to 3.73$)$ \\
\hline Q4 (most) & & 2.02 (1.36 to 2.68$)$ & $2.47(1.79$ to 3.14$)$ & & 19.9 (16.9 to 22.9$)$ & 15.5 (12.4 to 18.6$)$ & & 3.61 (2.55 to 4.67$)$ & 3.27 (2.19 to 4.35$)$ \\
\hline$P_{\text {trend }}$ & & & $<0.001$ & & & $<0.001$ & & & $<0.001$ \\
\hline$P_{\text {linearity }}$ & & & 0.001 & & & $<0.001$ & & & 0.38 \\
\hline
\end{tabular}

Model 1 is adjusted for age and wear time at the 3HC. Model 2 is adjusted for model 1 and height, weight, current wealth, smoking, alcohol intake, comorbidity (lifetime stroke, Ml, diabetes and/or cancer: yes/no), and calendar date on which the activity monitor was worn for the first time.

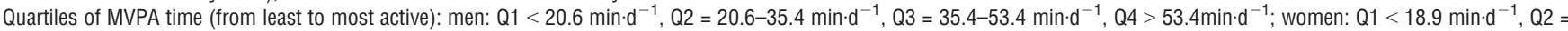
18.9-31.6 $\mathrm{min} \cdot \mathrm{d}^{-1}, \mathrm{Q} 3=31.6-47.2 \mathrm{~min} \cdot \mathrm{d}^{-1}, \mathrm{Q} 4>47.2 \mathrm{~min} \cdot \mathrm{d}^{-1}$.

who wore the monitor for shorter lengths of time were more likely to be older and have lower physical capability (see Document, Supplemental Digital Content, Table 2, Characteristics of men and women by average activity monitor wear time, http://links.lww.com/MSS/A583). Men accumulated $701 \mathrm{~min} \cdot \mathrm{d}^{-1}$ (SD, 76.5), and women, $669 \mathrm{~min} \cdot \mathrm{d}^{-1}$ (SD, 71.7) of sedentary time, whereas the mean amount of MVPA was $39 \mathrm{~min} \cdot \mathrm{d}^{-1}(\mathrm{SD}, 24.8)$ for men and $35 \mathrm{~min} \cdot \mathrm{d}^{-1}(\mathrm{SD}, 21.6)$ for women. There were moderate inverse correlations between sedentary time and MVPA for both sexes (spearman $\rho$ : men, -0.39 ; women, -0.42 ).

Participants who spent the most time in MVPA (Q4) had stronger grip strength, faster UWS, and faster TCSS than those who undertook the least MVPA (Q1) (Table 2). These differences persisted in multivariable models after adjustment for a range of anthropometric, health, and lifestyle confounders $\left(P_{\text {trend }}\right.$ all $\left.<0.001\right)$. There was consistent and strong evidence that associations between MVPA and UWS deviated from linearity $\left(P_{\text {linearity }}<0.001\right)$ (Table 2$)$. The largest differences in UWS were observed between the two lowest quartiles of MVPA in both sexes, with smaller differences between quartiles 2,3 , and 4 .

Participants who spent the shortest time sedentary (Q4) were stronger and had faster UWS and faster TCSS than those who were most sedentary (Q1) (Table 3). These findings persisted after adjustment for a range of confounders, although associations with grip strength and TCSS were weak in men (grip, $P_{\text {trend }}=0.03$; TCSS: $P_{\text {trend }}=0.21$ ). There was no convincing evidence that associations deviated from linearity (Table 3 ).

Although associations across both MVPA and sedentary time quartiles persisted after adjustment for potential confounders, these analyses do not address whether these associations are mutually independent. Therefore, MVPA and sedentary time were included together in multiple linear regression models, along with other covariables. Figures 1 and 2 show the mean adjusted physical capability of men and women by quartiles of MVPA and sedentary time, as estimated from the respective regression models. The previously observed strong associations between higher amounts

TABLE 3. Differences in physical capability by quartile of sedentary time.

\begin{tabular}{|c|c|c|c|c|c|c|c|c|c|}
\hline \multirow{2}{*}{$\begin{array}{l}\text { Sedentary Time } \\
\text { (Quartiles) }\end{array}$} & \multicolumn{3}{|c|}{ Difference in Grip Strength (95\% CI) (kg) } & \multicolumn{3}{|c|}{ Difference in UWS $(95 \% \mathrm{CI})\left(\mathrm{cm} \cdot \mathrm{s}^{-1}\right)$} & \multicolumn{3}{|c|}{ Difference in TCSS $(95 \% \mathrm{CI})$ (stands per minute) } \\
\hline & $n$ & Model 1 & Model 2 & $n$ & Model 1 & Model 2 & $n$ & Model 1 & Model 2 \\
\hline Men & 1664 & & & 1662 & & & 1506 & & \\
\hline Q1 (most) & & 0 & 0 & & 0 & 0 & & 0 & 0 \\
\hline Q2 & & $-0.30(-1.28$ to 0.68$)$ & $0.11(-0.85$ to 1.08$)$ & & 6.8 (3.7 to 9.9$)$ & $5.5(2.5$ to 8.6$)$ & & $1.36(0.21$ to 2.51$)$ & $1.12(-0.02$ to 2.25$)$ \\
\hline Q3 & & $1.00(-0.03$ to 2.02$)$ & $1.51(0.49$ to 2.52$)$ & & 7.9 (4.7 to 11.2$)$ & $6.3(3.1$ to 9.6$)$ & & $0.97(-0.23$ to 2.18$)$ & $0.80(-0.41$ to 2.01$)$ \\
\hline Q4 (least) & & $-0.01(-1.14$ to 1.12$)$ & $0.86(-0.28$ to 1.99$)$ & & $9.3(5.7$ to 12.9$)$ & 7.5 (3.8 to 11.1$)$ & & $1.25(-0.06$ to 2.57$)$ & $1.11(-0.23$ to 2.48$)$ \\
\hline$P_{\text {trend }}$ & & & 0.03 & & & $<0.001$ & & & 0.21 \\
\hline$P_{\text {linearity }}$ & & & 0.04 & & & 0.06 & & & 0.28 \\
\hline Women & 2022 & & & 2029 & & & 1834 & & \\
\hline Q1 (most) & & 0 & 0 & & 0 & 0 & & 0 & 0 \\
\hline Q2 & & $0.00(-0.62$ to 0.62$)$ & $0.26(-0.34$ to 0.86$)$ & & $5.6(2.7$ to 8.4$)$ & 4.6 (1.9 to 7.4$)$ & & $1.10(0.09$ to 2.10$)$ & $1.03(0.04$ to 2.01$)$ \\
\hline Q3 & & $0.69(0.05$ to 1.34$)$ & $1.05(0.41$ to 1.68$)$ & & 9.1 (6.1 to 12.1$)$ & 7.2 (4.3 to 10.1$)$ & & 1.53 (0.48 to 2.57$)$ & 1.28 (0.25 to 2.32$)$ \\
\hline Q4 (least) & & $0.83(0.11$ to 1.56$)$ & $1.44(0.72$ to 2.16$)$ & & $11.6(8.2$ to 14.9$)$ & $9.2(6.0$ to 12.6$)$ & & 2.21 (1.03 to 3.38$)$ & 1.85 (0.68 to 3.02$)$ \\
\hline$P_{\text {trend }}$ & & & $<0.001$ & & & $<0.001$ & & & 0.003 \\
\hline$P_{\text {linearity }}$ & & & 0.58 & & & 0.37 & & & 0.61 \\
\hline
\end{tabular}

Model 1 is adjusted for age and wear time at the $3 \mathrm{HC}$. Model 2 is adjusted for model 1 and height, weight, current wealth, smoking, alcohol intake, comorbidity (lifetime stroke, MI, diabetes, and/or cancer: yes/no), and calendar date on which the activity monitor was worn for the first time.

Quartiles of sedentary time (from most to least sedentary): men: Q1 $>12.3 \mathrm{~h} \cdot \mathrm{d}^{-1}, \mathrm{Q} 2=11.6-12.3 \mathrm{~h} \cdot \mathrm{d}^{-1}, \mathrm{Q} 3=10.9-11.6 \mathrm{~h} \cdot \mathrm{d}^{-1}, \mathrm{Q} 4<10.9 \mathrm{~h} \cdot \mathrm{d}^{-1} ;$ women: $\mathrm{Q} 1>11.8 \mathrm{~h} \cdot \mathrm{d}^{-1}, \mathrm{Q} 2=11.1-$ $11.8 \mathrm{~h} \cdot \mathrm{d}^{-1}, \mathrm{Q} 3=10.3-11.1 \mathrm{~h} \cdot \mathrm{d}^{-1}, \mathrm{Q} 4<10.3 \mathrm{~h} \cdot \mathrm{d}^{-1}$. 

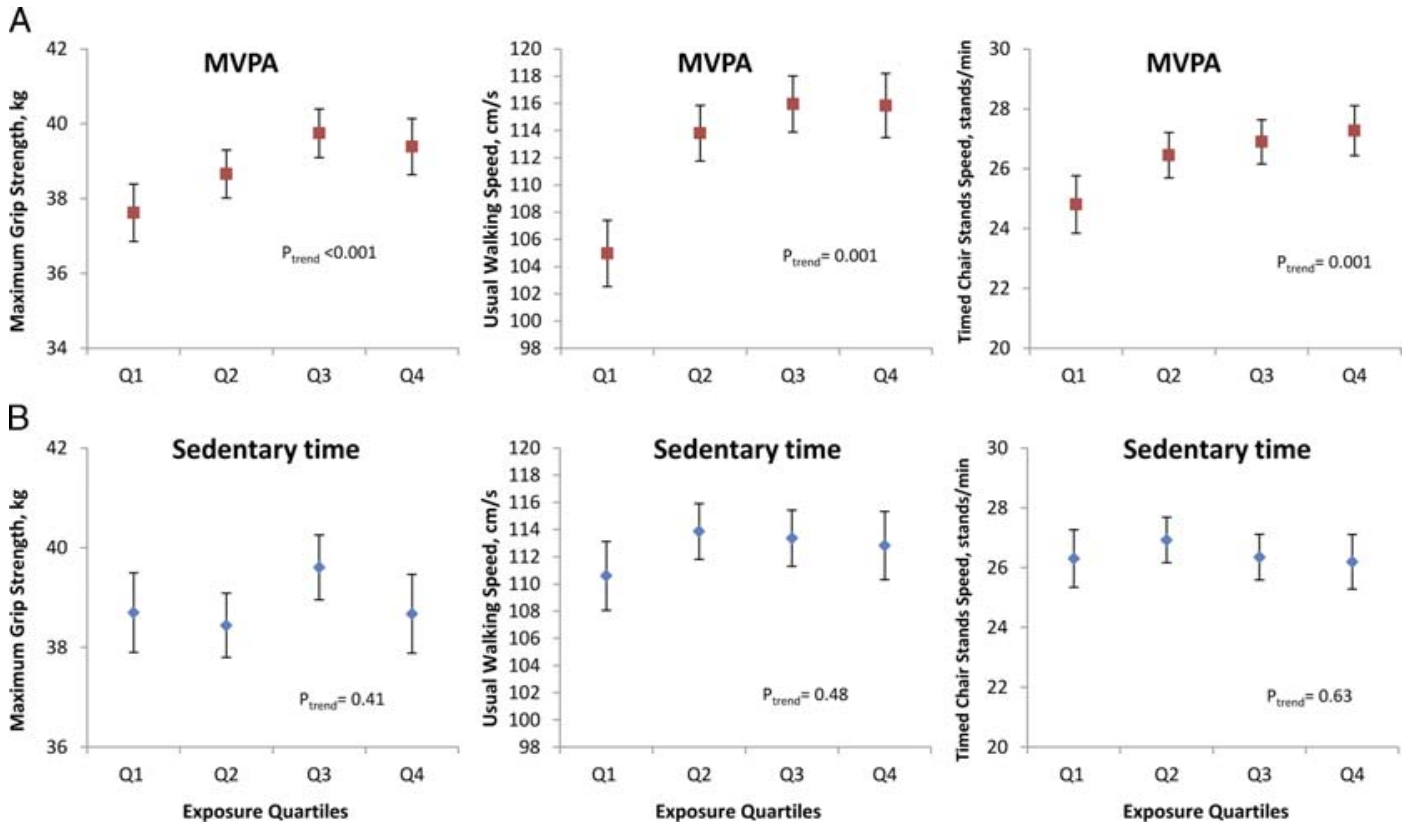

FIGURE 1-Associations between MVPA (A), sedentary time (B), and physical capability in men (all models were adjusted for age, height, weight, current wealth, smoking, alcohol intake, comorbidity, monitor wear time, calendar date on which the activity monitor was worn for the first time, and mutually for sedentary time and MVPA quartiles). Higher physical capability is associated with spending more time in MVPA (red squares) but not with spending less time sedentary (blue diamonds). As before, Q1 represents the lowest amount of MVPA but the highest amount of sedentary time.

of MVPA and stronger grip strength, faster UWS, and faster TCSS have persisted. However, the previously observed associations between lower amounts of sedentary time and higher physical capability have completely attenuated.

To explore possible interactions between MVPA and sedentary time, men and women were combined in analyses.
After stratification of the cohort by quartiles of MVPA (generated for the whole cohort), differences in physical capability associated with every extra hour of time spent sedentary were only evident within the lowest quartile of MVPA. In this quartile, every extra hour of sedentary time was associated with slower UWS $\left(-6.0 \mathrm{~cm} \cdot \mathrm{s}^{-1} ; 95 \% \mathrm{CI}\right.$,
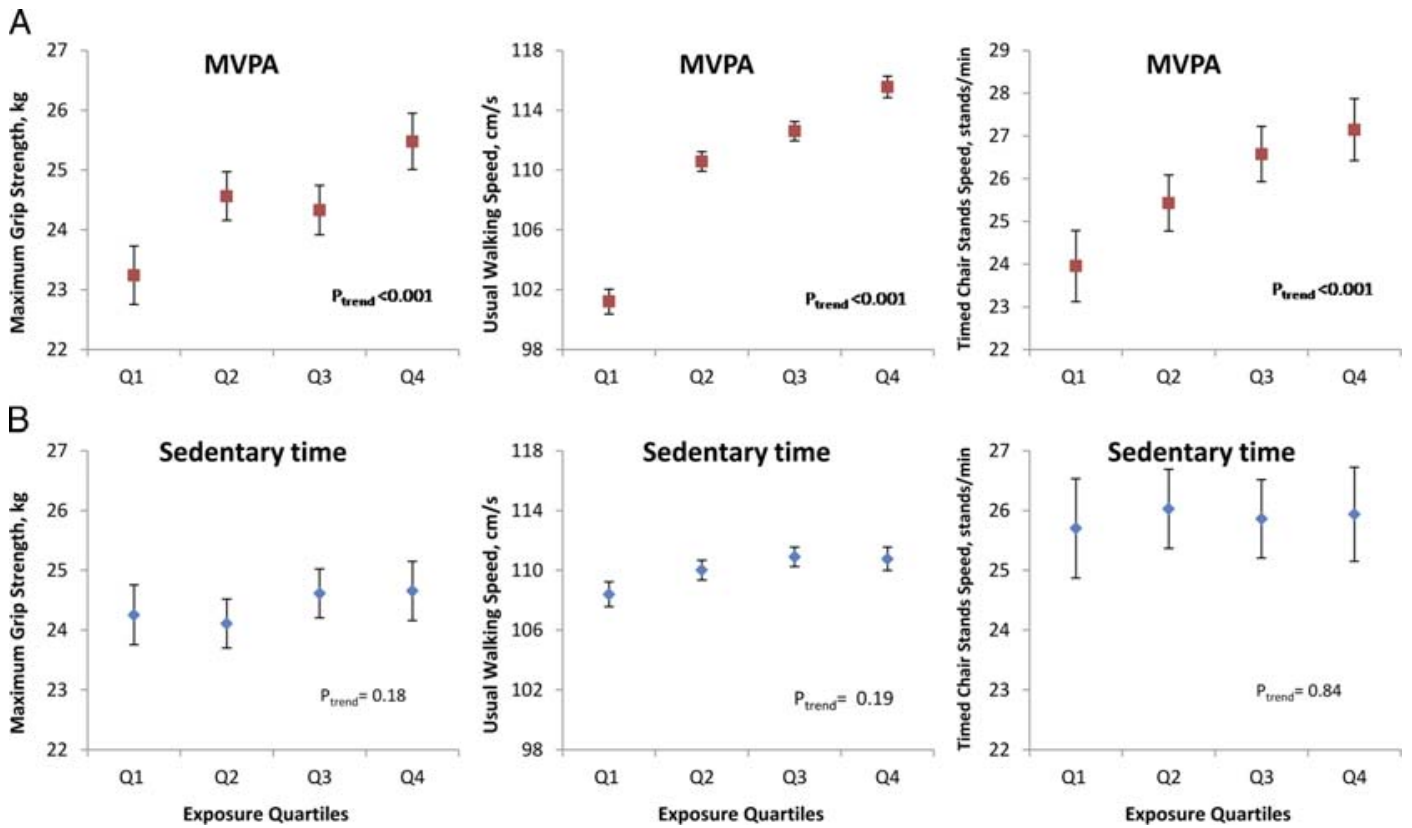

FIGURE 2-Associations between MVPA (A), sedentary time (B), and physical capability in women (all models were adjusted for age, height, weight, current wealth, smoking, alcohol intake, comorbidity, monitor wear time, calendar date on which the activity monitor was worn for the first time, and mutually for sedentary time and MVPA quartiles). Higher physical capability is associated with spending more time in MVPA (red squares) but not with spending less time sedentary (blue diamonds). As before, Q1 represents the lowest amount of MVPA but the highest amount of sedentary time. 
TABLE 4. Differences in physical capability per extra hour of daily sedentary time by quartile of MVPA.

\begin{tabular}{|c|c|c|c|c|}
\hline \multirow{2}{*}{$\begin{array}{l}\text { Physical Capability } \\
\text { Measure }\end{array}$} & \multicolumn{4}{|c|}{ MVPA Quartile $\left(\min \cdot d^{-1}\right)^{a}$} \\
\hline & $<19.5$ & $19.5-33.3$ & $33.3-50.3$ & $>50.3$ \\
\hline Grip (kg) & $\begin{array}{c}-0.79(-1.36 \text { to }-0.22) \\
P=0.01\end{array}$ & $\begin{array}{c}0.12(-0.50 \text { to } 0.74) \\
P=0.71\end{array}$ & $\begin{array}{c}0.32(-0.29 \text { to } 0.94) \\
P=0.31\end{array}$ & $\begin{array}{c}-0.14(-0.60 \text { to } 0.31) \\
P=0.54\end{array}$ \\
\hline UWS $\left(\mathrm{cm} \cdot \mathrm{s}^{-1}\right)$ & $\begin{array}{c}-5.4(-7.6 \text { to }-3.3) \\
P<0.001\end{array}$ & $\begin{array}{c}-0.75(-3.0 \text { to } 1.5) \\
P=0.51\end{array}$ & $\begin{array}{c}1.10(-1.0 \text { to } 3.3) \\
P=0.32\end{array}$ & $\begin{array}{c}-0.3(-2.0 \text { to } 1.3) \\
P=0.70\end{array}$ \\
\hline TCS (stands per minute) & $\begin{array}{c}-0.79(-1.53 \text { to }-0.05) \\
P=0.04\end{array}$ & $\begin{array}{c}0.17(-0.58 \text { to } 0.93) \\
P=0.65\end{array}$ & $\begin{array}{c}0.35(-0.51 \text { to } 1.21) \\
P=0.42\end{array}$ & $\begin{array}{c}0.01(-0.66 \text { to } 0.65) \\
P=0.98\end{array}$ \\
\hline
\end{tabular}

Values are adjusted for sex, age, height, weight, current wealth, smoking, alcohol intake, comorbidity (lifetime stroke, MI, diabetes, and/or cancer: yes/no) at the 3HC, monitor wear time, and calendar date on which the activity monitor was worn for the first time.

${ }^{a}$ MVPA quartiles characterized for the whole cohort (not sex specific). Numbers in MVPA quartiles: grip: Q1 916, Q2 922, Q3 924, Q4 924; UWS: Q1 914, Q2 925, Q3 923, Q4 929; TCS: Q1 702, Q2 853, Q3 882, Q4 903.

-8.2 to -3.8$)$, lower grip strength $(-0.81 \mathrm{~kg} ; 95 \% \mathrm{CI}$, -1.38 to -0.24$)$, and slower TCSS $(-0.77$ stands per minute, $95 \% \mathrm{CI},-1.51$ to -0.03$)$. However, no differences in physical capability with sedentary time were observed within quartiles 2, 3, and 4 of MVPA (Table 4). Residual confounding within the lowest quartile of MVPA may have explained the associations between sedentary time and physical capability, especially given the nonlinear relation between UWS and MVPA. Accordingly, every extra minute of MVPA within the lowest MVPA quartile was associated with stronger grip strength $(0.08 \mathrm{~kg} ; 95 \% \mathrm{CI},-0.01$ to $0.17)$, faster UWS $\left(1.5 \mathrm{~cm} \cdot \mathrm{s}^{-1} ; 95 \% \mathrm{CI}, 1.1-1.8\right)$, and faster TCSS (0.21 stands per minute; $95 \%$ CI, 0.09-0.33).

Multivariable fractional polynomial regression models including sedentary time $\left(\mathrm{h} \cdot \mathrm{d}^{-1}\right)$ and MVPA $\left(\min \cdot \mathrm{d}^{-1}\right)$, as well as all other covariables were constructed for each physical capability measure. There was no evidence of interaction between sedentary time and MVPA (UWS, $P_{\text {interaction }}=0.14$; grip, $P_{\text {interaction }}=0.69$; TCSS, $\left.P_{\text {interaction }}=0.76\right)$ with respect to any of the outcome measures. Furthermore, no associations between sedentary time and physical capability were evident after accounting for MVPA ( $P$ values all $>0.05$ ). In addition, no interactions or independent associations of sedentary time were evident if the cohort was stratified by age group ( $<70 \mathrm{vs} \geq 70 \mathrm{yr}$; results are available upon request).

Sensitivity analyses were performed using accelerometer data averaged over 60 -s epochs. In similarity with results from data in the form of 5-s epochs, 1654 men and 2039 women had four or more valid wear days (along with complete covariable data) and wear time was $864 \mathrm{~min} \cdot \mathrm{d}^{-1}$ $(\mathrm{SD}, 71)$ for men and $851 \mathrm{~min} \cdot \mathrm{d}^{-1}(\mathrm{SD}, 64)$ for women. However, estimates of sedentary time (men, $9.5 \mathrm{~h} \cdot \mathrm{d}^{-1}$ (SD, 1.4) or $572 \mathrm{~min} \cdot \mathrm{d}^{-1}$ (SD, 85); women, $8.9 \mathrm{~h} \cdot \mathrm{d}^{-1}(\mathrm{SD}, 1.4)$ or $532 \mathrm{~min} \cdot \mathrm{d}^{-1}$ (SD, 82)) and MVPA (men: $25 \mathrm{~min} \cdot \mathrm{d}^{-1}$ (SD, $22)$; women $20 \mathrm{~min}^{-1} \mathrm{~d}^{-1}(\mathrm{SD}, 18)$ ) were substantially lower. Sex-specific quartiles of sedentary time and MVPA were associated with each of the physical capability measures as before (data not shown), and, in mutually adjusted models, higher MVPA remained strongly associated with higher physical capability. However, associations between lower sedentary time and higher UWS did not completely attenuate after adjustment for MVPA (see Document, Supplemental Digital Content, Figures 1 and 2, http:// links.lww.com/MSS/A583). There was no evidence of interactions between sedentary time and MVPA with respect to any of the physical capability measures considered $(P$ values for interaction terms fitted within fractional polynomial models all remained $>0.05$ ).

\section{DISCUSSION}

This study reports cross-sectional associations between higher MVPA and higher physical capability in communitydwelling men and women age 48-92 yr. There was no evidence to suggest a threshold amount of MVPA below which more MVPA is not associated with higher physical capability; in fact, these data suggest the opposite effect, namely that increasing MVPA from Q1 to Q2, i.e., achieving greater than around $19 \mathrm{~min} \cdot \mathrm{d}^{-1}$, is associated with the greatest benefit for physical capability. In addition, within the lowest quartile of MVPA, even 1 min more per day of MVPA was associated with higher physical capability. This warrants further investigation in longitudinal and interventional studies. If low "doses" of MVPA could confer benefit, this would have important public health implications because many older adults are unable to meet current recommendations.

These findings also support other work associating higher amounts of MVPA with better physical capability (29) and add to the evidence that this association is strong and consistent when PA is measured objectively. Objective measurement of MVPA removes the measurement error associated with PAQ, attributable to reporting bias and difficulties with recall (17). Recall error may be especially problematic in older population groups because older adults tend to accumulate MVPA by engaging in short but regular activity episodes, which may be less memorable $(7,27)$, and older population groups are likely to have higher prevalence of conditions, which impair memory.

However, in contrast to a recent report in older Portuguese adults, associations between physical capability and sedentary time in this study were not independent of time spent in MVPA (32). The results presented here are also in contrast to findings from two other studies reporting independent associations between longer sedentary time and lower selfreported physical function $(2,12)$. One explanation for these disparities could be differences in the methods of adjustment for MVPA between studies. Others have stratified analyses 
by broad category of MVPA, e.g., "sufficient" versus "insufficient" (12), or it is not clear how MVPA has been adjusted for (32), leaving residual confounding a possibility. In analyses presented here, MVPA was accounted for using the same level of precision as sedentary time, e.g., quartiles of MVPA and quartiles of sedentary time. In addition, any time spent in MVPA was considered relevant, rather than only counting MVPA accumulated in 10-min bouts (12), and it was possible to differentiate periods of both MVPA and sedentary time with high resolution because of the analysis of data over 5-s epochs rather than the traditional 60 -s epoch. This is likely to lead to more accurate estimates of the PA exposures concerned. For example, shorter epoch length has been associated with higher estimates of total daily MVPA (26), which was also our finding in the sensitivity analyses conducted. This may reflect the short, sporadic bouts of MVPA accumulated in daily life, which may not be picked up when data is averaged over 60 -s intervals. It was also interesting to note that although estimates of both sedentary time and MVPA reduced when the data was analyzed with lower resolution, MVPA was affected to a greater extent than sedentary time. Estimates of MVPA were $30 \%-$ $40 \%$ lower, whereas estimates of sedentary time were only $20 \%$ lower, and this could explain why associations between sedentary time and physical capability, particularly UWS, were not completely attenuated by MVPA in the sensitivity analyses. Therefore, because the use of shorter epoch length is likely to be more accurate, the improved measurement of our exposures may explain disparities with previous study findings.

Although no independent associations between sedentary time and physical capability were identified in initial main analyses, it seemed as though there may be an interaction between sedentary time and MVPA. Every extra hour of sedentary time was associated with lower physical capability in those undertaking $\leq 19 \mathrm{~min} \cdot \mathrm{d}^{-1}$ of MVPA but not in those undertaking higher amounts of MVPA. However, considering the steep gradient of association between MVPA and physical capability across low durations, this could also be explained by residual confounding within the lowest MVPA quartile. If relations between MVPA and physical capability are not linear, different MVPA strata would account for MVPA with differing efficiency. Consistent with this, when MVPA and sedentary time were entered as continuous measures in models capable of accounting for nonlinear relations, no MVPA-sedentary time interactions or independent associations between physical capability and sedentary time were observed.

However, this does not preclude an association between sedentariness and physical capability. The accelerometer used in this study cannot differentiate standing still from sitting. The physiological and metabolic consequences of prolonged sitting $(1,40)$ are not associated with prolonged standing, and different health associations have been observed with standing compared with sitting. For example, prolonged sitting has been associated with higher mortality
(20), whereas prolonged standing has been associated with lower mortality (19).

In addition, we noted that monitor wear time varied considerably and those who wore the accelerometer for the shortest time were more likely to be women, older, and have low physical capability (see Document, Supplemental Digital Content, Table 2, Characteristics of men and women by average activity monitor wear time, http://links.lww.com/MSS/A583). For example, UWS was over $9 \mathrm{~cm} \cdot \mathrm{s}^{-1}$ slower in those who wore the monitor for the least time compared with those who wore the monitor for the most time. It is likely that differences in sleep patterns explain some of these observations (23), but we cannot exclude the possibility that monitors were taken off earlier in those with lower physical capability for other reasons. This would mostly likely affect the accuracy of sedentary time measurement, given that the largest proportion of wear time was spent sedentary and it is unlikely that participants would remove the monitor during periods of MVPA (with the exception of waterbased activity).

This study, as with other reports examining objectively measured PA and physical capability to date, is limited by its cross-sectional design restricting the ability to make causal inferences. Although evidence from longitudinal studies (29), intervention studies (30), and physiological studies $(11,35)$ suggests that PA may lead to improved physical performance, low physical capability may also be a determinant of low activity participation (5). The most likely true scenario is that a bidirectional, vicious cycle exists between low PA and low physical capability, and perhaps this could explain the steeper cross-sectional associations between MVPA and physical capability at lower levels of MVPA.

Other limitations to our study include the insensitivity of accelerometers to accurately capture certain types of exercise, e.g., cycling and swimming, and their inability to differentiate resistance exercise from aerobic activity. Associations between aerobic activity and physical function in later life are less clear (8) than the well-established benefits of resistance exercise training. Furthermore, the participants in EPIC-Norfolk are almost exclusively Caucasian and, although comparable with a nationally representative sample at recruitment, generalizability is limited by the selective attrition of more vulnerable cohort members over time (15). Finally, our analyses could not account for the presence of arthritis, a condition prevalent in later life, which may both limit activity participation and contribute to low physical capability. The absence of knowledge about this potentially important confounding variable limits our interpretation of the associations observed. Nevertheless, the data presented represents a large number of community-based men and women with objective measurement of sedentary time, MVPA, and physical capability, enabling cautious conclusions to be drawn on dose-response relations.

In summary, we found strong associations between higher physical capability and higher MVPA but no evidence of 
independent associations with objectively measured sedentary time.

The EPIC-Norfolk study would not be possible without our participants, the general practitioners of Norfolk, or the many administrative and research staff who have worked on the study over its duration. We thank all of these people for their cooperation and support.

V. L. K. declares a Wellcome Trust clinical training fellowship (092077/Z/10/Z), and K. W., a British Heart Foundation basic science research fellowship (FS/12/58/29709). A. J. M. C., N. J. W., and S. B. are supported by MRC program grants (MC_UU_12015/3

\section{REFERENCES}

1. Bey L, Hamilton MT. Suppression of skeletal muscle lipoprotein lipase activity during physical inactivity: a molecular reason to maintain daily low-intensity activity. $J$ Physiol. 2003;551(Pt 2):673-82.

2. Buman MP, Hekler EB, Haskell WL, et al. Objective lightintensity physical activity associations with rated health in older adults. Am J Epidemiol. 2010;172(10):1155-65.

3. Cooper R, Kuh D, Hardy R. Objectively measured physical capability levels and mortality: systematic review and meta-analysis. BMJ. 2010;341:c4467.

4. Cooper R, Mishra GD, Kuh D. Physical activity across adulthood and physical performance in midlife: findings from a British birth cohort. Am J Prev Med. 2011;41(4):376-84.

5. Crombie IK, Irvine L, Williams B, et al. Why older people do not participate in leisure time physical activity: a survey of activity levels, beliefs and deterrents. Age Ageing. 2004;33(3):287-92.

6. Daly RM, Ahlborg HG, Ringsberg K, Gardsell P, Sernbo I, Karlsson MK. Association between changes in habitual physical activity and changes in bone density, muscle strength, and functional performance in elderly men and women. J Am Geriatr Soc. 2008;56(12): 2252-60.

7. Davis MG, Fox KR, Hillsdon M, Sharp DJ, Coulson JC, Thompson JL. Objectively measured physical activity in a diverse sample of older urban UK adults. Med Sci Sports Exerc. 2011;43(4):647-54.

8. Denison HJ, Syddall HE, Dodds R, et al. Effects of aerobic exercise on muscle strength and physical performance in communitydwelling older people from the Hertfordshire cohort study: a randomized controlled trial. J Am Geriatr Soc. 2013;61(6):1034-6.

9. Department of Health. Older adults. In: Start Active, Stay Active: a Report on Physical Activity from the Four Home Countries' Chief Medical Officers. London (United Kingdom): Department of Health, Physical Activity, Health Improvement and Protection; 2011 p. 38-44.

10. Freedson PS, Melanson E, Sirard J. Calibration of the Computer Science and Applications, Inc. accelerometer. Med Sci Sports Exerc. 1998;30(5):777-81.

11. Fujita $\mathrm{S}, \mathrm{Abe} \mathrm{T}$, Drummond $\mathrm{MJ}$, et al. Blood flow restriction during low-intensity resistance exercise increases S6K1 phosphorylation and muscle protein synthesis. J Appl Physiol (1985). 2007;103(3):903-10.

12. Gennuso KP, Gangnon RE, Matthews CE, Thraen-Borowski KM, Colbert LH. Sedentary behavior, physical activity, and markers of health in older adults. Med Sci Sports Exerc. 2013;45(8):1493-500.

13. Guralnik JM, Ferrucci L, Simonsick EM, Salive ME, Wallace RB. Lower-extremity function in persons over the age of 70 years as a predictor of subsequent disability. N Engl J Med. 1995;332(9):556-61.

14. Hamer M, Stamatakis E. Screen-based sedentary behavior, physical activity, and muscle strength in the English longitudinal study of ageing. PLoS One. 2013;8(6):e66222.

15. Hayat SA, Luben R, Keevil VL, et al. Cohort profile: A prospective cohort study of objective physical and cognitive capability and visual health in an ageing population of men and women in Norfolk (EPIC-Norfolk 3). Int J Epidemiol. 2014;43(4):1063-72. and MC_UU_12015/4). The EPIC-Norfolk study was supported by program grants from the Medical Research Council (G9502233; G0401527) and Cancer Research United Kingdom (C864/A8257). A grant from Research into Ageing (262) funded the $3 \mathrm{HC}$ clinic.

V. L. K. analyzed and interpreted the data and drafted the manuscript with contributions from A. J. M. C., K. W., K. T. K., and S. B. K. T. K., S. B., N. J. W. and R. L. contributed to study concepts and design, and K. T. K., S. B., N. J. W., and R. L. contributed to participant recruitment, data collection, and management.

No conflicts of interest were declared by any authors.

The results of the present study do not constitute endorsement by the American College of Sports Medicine.

16. Healy GN, Clark BK, Winkler EA, Gardiner PA, Brown WJ, Matthews CE. Measurement of adults' sedentary time in populationbased studies. Am J Prev Med. 2011;41(2):216-27.

17. Helmerhorst HJ, Brage S, Warren J, Besson H, Ekelund U. A systematic review of reliability and objective criterion-related validity of physical activity questionnaires. Int J Behav Nutr Phys Act. 2012;9:103.

18. Jeon CY, Lokken RP, Hu FB, van Dam RM. Physical activity of moderate intensity and risk of type 2 diabetes: a systematic review. Diabetes Care. 2007;30(3):744-52.

19. Katzmarzyk PT. Standing and mortality in a prospective cohort of Canadian adults. Med Sci Sports Exerc. 2014;46(5):940-6.

20. Katzmarzyk PT, Church TS, Craig CL, Bouchard C. Sitting time and mortality from all causes, cardiovascular disease, and cancer. Med Sci Sports Exerc. 2009;41(5):998-1005.

21. Keevil VL, Wijndaele K, Luben R, Sayer AA, Wareham NJ, Khaw KT. Television viewing, walking speed, and grip strength in a prospective cohort study. Med Sci Sports Exerc. 2015;47(4):735-42.

22. Khaw KT, Jakes R, Bingham S, et al. Work and leisure time physical activity assessed using a simple, pragmatic, validated questionnaire and incident cardiovascular disease and all-cause mortality in men and women: the European Prospective Investigation into Cancer in Norfolk prospective population study. Int $J$ Epidemiol. 2006;35(4):1034-43.

23. Leng Y, Wainwright NW, Cappuccio FP, et al. Self-reported sleep patterns in a British population cohort. Sleep Med. 2014;15(3): 295-302.

24. Manini TM, Everhart JE, Patel KV, et al. Activity energy expenditure and mobility limitation in older adults: differential associations by sex. Am J Epidemiol. 2009;169(12):1507-16.

25. Martin HJ, Syddall HE, Dennison EM, Cooper C, Sayer AA. Relationship between customary physical activity, muscle strength and physical performance in older men and women: findings from the Hertfordshire Cohort Study. Age Ageing. 2008;37(5):589-93.

26. Orme M, Wijndaele K, Sharp SJ, Westgate K, Ekelund U, Brage S. Combined influence of epoch length, cut-point and bout duration on accelerometry-derived physical activity. Int J Behav Nutr Phys Act. 2014;11(1):34.

27. Ortlieb S, Dias A, Gorzelniak L, et al. Exploring patterns of accelerometry-assessed physical activity in elderly people. Int $J$ Behav Nutr Phys Act. 2014;11(1):28.

28. Patel KV, Coppin AK, Manini TM, et al. Midlife physical activity and mobility in older age: the InCHIANTI study. Am J Prev Med. 2006;31(3):217-24.

29. Paterson DH, Warburton DE. Physical activity and functional limitations in older adults: a systematic review related to Canada's Physical Activity Guidelines. Int J Behav Nutr Phys Act. 2010;7:38.

30. Peterson MD, Rhea MR, Sen A, Gordon PM. Resistance exercise for muscular strength in older adults: a meta-analysis. Ageing Res Rev. 2010;9(3):226-37.

31. Royston P, Sauerbrei W. Continuous-by-continuous interactions. In: Multivariable Model Building: a Pragmatic Approach to 
Regression Anaylsis Based on Fractional Polynomials for Modelling Continuous Variables. Wiley; 2008 p. 172-80.

32. Santos DA, Silva AM, Baptista F, et al. Sedentary behavior and physical activity are independently related to functional fitness in older adults. Exp Gerontol. 2012;47(12):908-12.

33. Simonsick EM, Guralnik JM, Volpato S, Balfour J, Fried LP. Just get out the door! Importance of walking outside the home for maintaining mobility: findings from the women's health and aging study. J Am Geriatr Soc. 2005;53(2):198-203.

34. Sofi F, Capalbo A, Cesari F, Abbate R, Gensini GF. Physical activity during leisure time and primary prevention of coronary heart disease: an updated meta-analysis of cohort studies. Eur $J$ Cardiovasc Prev Rehabil. 2008;15(3):247-57.

35. Starkie R, Ostrowski SR, Jauffred S, Febbraio M, Pedersen BK. Exercise and IL-6 infusion inhibit endotoxin-induced TNF-alpha production in humans. FASEB J. 2003;17(8):884-6.
36. Togo F, Watanabe E, Park H, Shephard RJ, Aoyagi Y. Meteorology and the physical activity of the elderly: the Nakanojo Study. Int J Biometeorol. 2005;50(2):83-9.

37. Tolea MI, Costa PT, Terracciano A, et al. Sex-specific correlates of walking speed in a wide age-ranged population. $J$ Gerontol B Psychol Sci Soc Sci. 2010;65B(2):174-84.

38. Tucker JM, Welk GJ, Beyler NK. Physical activity in U.S.: adults compliance with the Physical Activity Guidelines for Americans. Am J Prev Med. 2011;40(4):454-61.

39. Visser M, Pluijm SM, Stel VS, Bosscher RJ, Deeg DJ. Physical activity as a determinant of change in mobility performance: the Longitudinal Aging Study Amsterdam. J Am Geriatr Soc. 2002;50(11): 1774-81.

40. Zderic TW, Hamilton MT. Physical inactivity amplifies the sensitivity of skeletal muscle to the lipid-induced downregulation of lipoprotein lipase activity. J Appl Physiol (1985). 2006;100(1):249-57. 